\title{
Universiteit
}

Leiden

The Netherlands

\section{Cognitive therapy does not prevent a response to tryptophan depletion in patients also treated with antidepressants}

Does, A.J.W. van der; Booij, L.

\section{Citation}

Does, A. J. W. van der, \& Booij, L. (2005). Cognitive therapy does not prevent a response to tryptophan depletion in patients also treated with antidepressants. Biological Psychiatry, 58, 913-915. Retrieved from https://hdl.handle.net/1887/14417

Version: $\quad$ Not Applicable (or Unknown)

License: $\quad$ Leiden University Non-exclusive license

Downloaded from: $\quad$ https://hdl.handle.net/1887/14417

Note: To cite this publication please use the final published version (if applicable). 


\title{
Cognitive Therapy Does Not Prevent a Response to Tryptophan Depletion in Patients also Treated with Antidepressants
}

\author{
A.J. Willem Van der Does and Linda Booij
}

Background: Acute tryptophan depletion (ATD) induces depressive symptoms in remitted depressed patients treated with serotonergic medications, but not in patients treated with noradrenergic medications or electroconvulsive therapy. A recent study suggests that cognitive therapy (CT) protects against the effects of ATD, but the evidence is questionable. The present study compared the effect of ATD in patients who were treated with antidepressant medication and CT $(\mathrm{n}=17)$ versus antidepressant medication alone $(\mathrm{n}=23)$ during their latest episode.

Methods: Forty remitted depressed patients underwent high-dose and low-dose ATD in a randomized double-blind crossover design. Results: There were no differences in response to ATD between treatment groups. This applied to groups defined by lifetime and by recent CT experience.

Conclusions: Cognitive therapy does not protect against the effects of rapidly lowered plasma tryptophan levels in remitted depressed patients who are also treated with antidepressant medication.

\section{Key Words: Depression, tryptophan depletion, cognitive therapy, serotonin, antidepressants}

\section{A} cute tryptophan depletion (ATD) is a serotonergic challenge test that involves the rapid lowering of tryptophan plasma levels (Young et al 1985). It has been demonstrated that ATD induces depressive symptoms in patients treated with a selective serotonin reuptake inhibitor (SSRI), whereas patients treated with electroconvulsive therapy, noradrenergic antidepressants, or sleep deprivation are less likely to respond (Booij et al 2003; Van der Does 2001). Recently, O'Reardon et al (2004) compared the effect of ATD in remitted depressed patients treated with cognitive therapy (CT) versus an SSRI. Significant differences between active and "sham" depletion were found in the SSRI group only. The authors conclude that the therapeutic response to CT does not depend on an intact serotonin system. Since an earlier study has shown that unmedicated recovered depressed patients respond to ATD (Smith et al 1997), it might be concluded that CT protects against the depression-inducing effects of rapidly lowered tryptophan levels.

This conclusion, however, might be premature. Firstly, patients had not been randomized over treatment conditions before the ATD experiment. Consequently, there might have been other between-group differences that are known to be related to ATD response (e.g., past suicidality or chronicity; Booij et al 2002). Chronicity was not reported, but patients in the SSRI group were considerably older $(+17.6$ years; $p<.001)$. Secondly, there were only 10 patients per group, and in the CT group, the change of Hamilton scores after ATD was 5 points. Although this was less than the change in the SSRI group (8.2), it is actually not a negligible effect when compared with the ATD literature (Booij

From the Department of Psychology (AJWV, LB), Leiden University; and the Department of Psychiatry (AJWV), Leiden University Medical Center, Leiden, The Netherlands.

Address reprint requests to A.J.W. Van der Does, Ph.D., Department of Psychology, Leiden University, Wassenaarseweg 52, 2333 AK Leiden, The Netherlands; E-mail: vanderdoes@fsw.leidenuniv.nl.

Received November 19, 2004; revised April 25, 2005; accepted April 28, 2005. et al, in press). Thirdly, although the change in the CT group was not significantly higher than during "sham" depletion (5 vs. 3.2), this might have been due to a relatively large response during sham depletion and lack of statistical power, rather than true lack of response.

No other ATD study investigated CT-treated patients; however, some circumstantial evidence supports the conclusion of O'Reardon et al (2004). We recently found that high "cognitive reactivity" scores in SSRI-treated patients predict a larger response to ATD (Van der Does et al 2004). Cognitive reactivity is a psychological vulnerability marker of depression and is defined as the ease with which dysfunctional cognitions are triggered by mild (nonpathological) mood deteriorations. Cognitive reactivity scores are higher in remitted depressed patients treated with medications than in patients treated with CT and predict recurrences (Segal et al 1999). The combination of these findings suggests that CT-treated patients would be expected to have lower cognitive reactivity scores and to be less vulnerable to ATD. Because of the limitations of the O'Reardon study, however, more data are needed before it may be concluded that CT protects against the effects of depleted serotonin levels. The aim of the present report is to provide these data. Unlike O'Reardon et al (2004), who compared groups treated with either medication or CT, our design permitted comparison of combined treatment (medication $+\mathrm{CT}$ ) versus medication only. If CT makes patients resistant to ATD, the depressive response should be smaller in the combined treatment group.

\section{Methods and Materials}

\section{Patients and Procedures}

Forty-two patients underwent high-dose and low-dose ATD (102.5 vs. $25.7 \mathrm{~g}$ amino acids) in a double-blind randomized crossover design. Inclusion criteria were: age between 18 and 65 years, ongoing treatment with an SSRI or serotonin-noradrenalin reuptake inhibitor (SNRI) for at least 4 weeks, meeting DSM-IV criteria for depression in remission, and a (17-item version) Hamilton Depression Rating Scale (HRSD-17) score lower than 15 (cf. Frank et al 1991). Exclusion criteria were: substance abuse within last three months, psychosis (lifetime), major physical illness, and lactation and pregnancy. Diagnoses and demographic and clinical background data were collected with the 
Table 1. Sample Characteristics and Effects of Tryptophan Depletion in Patients Treated with Antidepressants or Combined Treatment (during latest episode)

\begin{tabular}{|c|c|c|c|c|c|}
\hline Variable & \multicolumn{2}{|c|}{ AD Group } & \multicolumn{2}{|c|}{$A D+C T$ Group } & Statistics \\
\hline Number of CT sessions (SD) & \multicolumn{2}{|c|}{$\mathrm{N} / \mathrm{A}$} & \multicolumn{2}{|c|}{$16.8(9.7)$} & N/A \\
\hline Gender (male/female) & \multicolumn{2}{|c|}{$13 / 10$} & \multicolumn{2}{|c|}{$7 / 10$} & $\chi^{2}=.92, p=.34$ \\
\hline Mean age (SD) & \multicolumn{2}{|c|}{$48.5(9.9)$} & \multicolumn{2}{|c|}{$42.2(9.1)$} & $F(1,38)=4.33, p=.04$ \\
\hline Mean number of episodes (SD) & \multicolumn{2}{|c|}{$4.8(6.1)$} & \multicolumn{2}{|c|}{$3.6(3.8)$} & $F(1,38)=.50, p=.48$ \\
\hline Single vs. recurrent past episodes & \multicolumn{2}{|c|}{$8 / 15$} & \multicolumn{2}{|c|}{$5 / 12$} & $\chi^{2}=.13, p=.72$ \\
\hline Mean remission duration, months (SD) & \multicolumn{2}{|c|}{$21.3(6.1)$} & \multicolumn{2}{|c|}{$4.2(2.8)$} & $F(1,38)=5.74, p=.02$ \\
\hline Remission status (partial vs. full) & \multicolumn{2}{|c|}{$16 / 7$} & \multicolumn{2}{|c|}{$13 / 4$} & $\chi^{2}=.23, p=.63$ \\
\hline Type of medication (SSRI/SNRI) & \multicolumn{2}{|c|}{$15 / 8$} & \multicolumn{2}{|c|}{$14 / 3$} & $\chi^{2}=1.44, p=.23$ \\
\hline Reduction (\%) of total Trp (SE) & \multicolumn{2}{|c|}{$-87.1(.9)$} & \multicolumn{2}{|c|}{$-85.1(1.2)$} & $F(1,37)=1.84, p=.18$ \\
\hline Reduction (\%) of Trp/LNAA (SE) & & & & & $F(1,27)=.24, p=.62$ \\
\hline \multirow[t]{2}{*}{ Number of responders to ATD } & \multicolumn{2}{|c|}{$\begin{array}{c}-93.2(.7) \\
9\end{array}$} & \multicolumn{2}{|c|}{8} & $\chi^{2}=.62, p=.43$ \\
\hline & Baseline & $+6.5 \mathrm{~h}$ & Baseline & $+6.5 \mathrm{~h}$ & Intervention by Time by Group \\
\hline MADRS - high dose ATD (SE) & $4.6(.9)$ & $8.6(1.5)$ & $4.8(.9)$ & $9.6(1.6)$ & $F(2,76)=.05, p=.95$ \\
\hline MADRS - low dose ATD (SE) & $3.3(.6)$ & $3.1(.8)$ & $6.3(1.2)$ & $6.7(1.0)$ & \\
\hline HRSD (SE) - high dose ATD (SE) & $2.9(.4)$ & $4.4(.7)$ & $2.5(.8)$ & $5.0(.9)$ & $F(2,76)=.11, p=.89$ \\
\hline HRSD (SE) - low dose ATD (SE) & $2.43(.4)$ & $2.0(.3)$ & $3.0(.5)$ & $3.3(.5)$ & \\
\hline BDI-II total (SE) - high dose ATD (SE) & $6.0(1.1)$ & $8.3(1.5)$ & $6.5(1.3)$ & $7.8(1.7)$ & $F(2,74)=.55, p=.58$ \\
\hline BDI-II total (SE) - low dose ATD (SE) & $6.4(1.4)$ & $6.0(1.4)$ & $8.1(1.6)$ & $6.6(1.5)$ & \\
\hline \multicolumn{6}{|l|}{ Comorbidity } \\
\hline None & \multicolumn{2}{|c|}{16} & \multicolumn{2}{|c|}{11} & $\chi^{2}=.10, p=.75$ \\
\hline Anorexia nervosa lifetime & \multicolumn{2}{|c|}{0} & \multicolumn{2}{|c|}{1} & \\
\hline Bulimia nervosa current & \multicolumn{2}{|c|}{1} & \multicolumn{2}{|c|}{0} & \\
\hline OCD lifetime & \multicolumn{2}{|c|}{1} & \multicolumn{2}{|c|}{1} & \\
\hline Panic disorder lifetime & \multicolumn{2}{|c|}{4 ( 2 current) } & & & \\
\hline Social anxiety disorder lifetime & & current) & & & \\
\hline
\end{tabular}

Biochemical change scores are based on $\mathrm{t}_{+6.5}$ Vs. $\mathrm{t}_{-1}$ during high-dose acute tryptophan depletion (ATD). Intervention by time interaction: $[F(2,76)=$ $10.45, p<.001$ (MADRS); $F(2,76)=8.65, p<.001$ (HRSD); $F(2,74)=4.46, p=.01$ (BDI-II). Statistics are from analyses without covariates.

$A D$, antidepressant; $C T$, cognitive therapy; SSRI, selective serotonin reuptake inhibitor; SNRI, serotonin-noradrenalin reuptake inhibitor; Trp, tryptophan; LNAA, large neutral amino acids; MADRS, Montgomery Asberg Depression Rating Scale; HRSD, Hamilton Rating Scale for Depression; BDI-II, Beck Depression Inventory - 2nd edition; OCD, obsessive compulsive disorder.

Structured Clinical Interview for DSM-IV (SCID-I) (First et al 1995) and checked in the medical records. The study was approved by an independent medical ethics committee, and all participants provided written informed consent. High- and lowdose ATD were designed to lower plasma tryptophan by $90 \%$ and 50\%, respectively. Other findings from subsamples have been reported elsewhere (Booij et al 2005; Booij et al, in press).

\section{Prior Treatment}

All participants were receiving ongoing treatment with either an SSRI $(n=30)$ or SNRI $(n=12)$. Eighteen patients had also received CT for their latest episode, either in group $(n=8)$ or individual format $(n=10)$. The time between the latest CT session and ATD varied between 0 and 4 weeks. Another 6 patients had been treated with CT during an earlier episode. Information about duration and format of CT was obtained in a clinical interview and checked with the therapists and the medical records.

\section{Behavioral Observations and Ratings}

Symptoms were assessed 1 hour before ATD $\left(t_{-1}\right), 6.5$ hours later $(\mathrm{t}+6.5)$, and the next morning $(\mathrm{t}+24)$ with the HRSD-17 (Hamilton 1960), the Montgomery-Asberg Depression Rating Scale (MADRS) (Montgomery and Asberg 1979), and the Beck Depression Inventory-II (BDI-II) (Beck et al 1996).

\section{Statistics}

Baseline group differences were examined with univariate analysis of variance and $\chi^{2}$ statistics. The effects of ATD were analyzed with repeated measures analysis of variance with General Linear Models. Within-subjects factors were intervention (low-dose vs. high-dose ATD) and time $\left(\mathrm{t}_{-1}, \mathrm{t}_{+6.5}, \mathrm{t}_{+24}\right)$; the between-subjects factor was group (antidepressants vs. antidepressants $+\mathrm{CT}$ ). Analyses were rerun, including covariates, to correct for any group differences on clinical or demographic characteristics.

\section{Results}

Two patients fulfilled inclusion and exclusion criteria at intake and during low-dose ATD, but had too-high MADRS scores on the morning of the high-dose session and were excluded. Results of the remaining sample are presented in Table 1. Differences in duration of remission were entirely due to four patients in the antidepressant-group who reached remission 6 to 7 years ago. There were no differences in remission duration when these four patients were omitted.

Acute tryptophan depletion induced the expected reductions of plasma tryptophan concentrations, and depressive symptoms 6.5 hours after high-dose but not after low-dose ATD. Seventeen patients (42.5\%) were responders to ATD (defined as an increase of MADRS or HRSD of at least 6 points; Booij et al 2005). Depressive symptoms had returned to baseline levels the next morning. There were no differences in ATD response on any symptom scale between treatment groups (statistics shown in Table 1). This was also true when the combined group was defined on the basis of lifetime CT experience ( $n=24)$ (all $p>$ 
.35). A third analysis involving only patients who had recently taken a CT module as part of a 16-week intensive (12 hours/ week) semi-residential program $(n=7)$ versus those who had never received CT $(n=16)$ also showed no differences (all $p>$ .58). Several analyses were conducted to control for possible confounders. Results were very similar when duration of remission and/or age were entered as covariates. The same was true when the four patients with long remission durations and/or patients on SNRIs were left out from analyses (with and without covariates). The CT format (individual, group) also did not affect the results.

\section{Discussion}

The present results do not support the position that CT improves biological vulnerability. This finding might seem at odds with O'Reardon et al (2004), but it should be noted that there are several differences between the two studies, the most important one being the fact that our CT group was also treated with antidepressants. The mechanism responsible for recovery in a combined treatment sample is uncertain. It may be possible that medications were solely responsible for recovery in this sample, rendering these patients vulnerable to ATD, whereas in O'Reardon et al (2004), one group had CT only. Patients in the present study who received intensive CT, however, were as likely to respond to ATD.

The present finding might seem difficult to reconcile with our previous finding that cognitive reactivity strongly predicts ATD response (Van der Does et al 2004). This can only be understood if one assumes that CT in combination with antidepressantseven when administered in a 16-week intensive program-does not necessarily lead to improved cognitive vulnerability scores. If this is true, then CT alone might lead to both improved biological vulnerability scores (smaller response to ATD; O'Reardon et al 2004) and improved cognitive vulnerability scores (lower cognitive reactivity; Segal et al 1999), although combined treatment does not have this effect. This implies that the protective effect of CT for recurrences of depressive episodes (Hollon et al 2005) might be reduced when given in combination with antidepressants. As noted in the introduction, however, it may be doubted whether the CT group in the O'Reardon study was not affected by ATD, so this conclusion remains speculative.

Future studies might investigate biological and psychological vulnerability in patients treated with CT, medications, combined treatment, and possibly other treatments. A new maintenance treatment - mindfulness-based CT-protects better against recurrences than CT alone (Ma and Teasdale 2004; Teasdale et al 2000) and also has a positive effect on a psychological vulnerability marker (Williams et al 2000). It would be interesting to see whether this treatment also affects biological vulnerability markers.

Beck AT, Steer RA, Brown GK (1996) Manual for the Beck Depression Inventory II. San Antonio, Texas: The Psychological Corporation.

Booij L, Van der Does AJW, Benkelfat C, Bremner JD, Cowen PJ, Fava M, et al (2002): Predictors of mood response to acute tryptophan depletion. A reanalysis. Neuropsychopharmacol 27:852-861.

Booij L, Van der Does AJW, Haffmans PMJ, Riedel WJ, Fekkes D, Blom MJB (2005): The effects of high and low dose tryptophan depletion on mood and cognitive functions of remitted depressed patients. $J$ Psychopharmacol 19:267-276.

Booij L, Van der Does AJW, Haffmans PJ, Spinhoven Ph, McNally RJ (in press): Acute Tryptophan Depletion as a model of depressive relapse: Behavioral specificity and ethical considerations. Br J Psychiatry.

Booij L, Van der Does AJW, Riedel WJ (2003): Monoamine depletion in psychiatric and healthy populations: Review. Mol Psychiatry 8:951-973.

First MB, Spitzer RL, Gibbon M, Williams JBW(1995): Structured Clinical Interview for DSM-IV Axis I Disorders. (SCID-I/P). New York: Biometrics Research Department, NYSPI.

Frank E, Prien RF, Jarrett RB, Keller MB, Kupfer DJ, Lavori PW, et al (1991): Conceptualization and rationale for consensus definitions of terms in major depressive disorder - remission, recovery, relapse, and recurrence. Arch Gen Psychiatry 48:851-855.

Hamilton M (1960): A rating scale for depression. J Neurol Neurosurg Psychiatry 23:56-62.

Hollon SD, DeRubeis RJ, Shelton, RC, Amsterdam JD, Salomon RM, O'Reardon JP, et al (2005): Prevention of relapse following cognitive therapy versus medications in moderate to severe depression. Arch Gen Psychiatry 62:417-422.

Ma SH, Teasdale JD (2004): Mindfulness-based cognitive therapy for depression: Replication and exploration of differential relapse prevention effects. J Cons Clin Psychol 72:31-40.

Montgomery SA, Asberg M (1979): A new depression scale designed to be sensitive to change. Br J Psychiatry 134:382-389.

O'Reardon JP, Chopra MP, Bergan A, Gallop R, DeRubeis RJ, Crits-Christoph P (2004): Response to tryptophan depletion in major depression treated with either cognitive therapy or selective serotonin reuptake inhibitor antidepressants. Biol Psychiatry 55:957-959.

Segal ZV, Gemar M, Williams S (1999): Differential cognitive response to a mood challenge following successful cognitive therapy or pharmacotherapy for unipolar depression. J Abn Psychol 108:3-10.

Smith KA, Fairburn CG, Cowen PJ (1997): Relapse of depression after rapid depletion of tryptophan. Lancet 349:915-919.

Teasdale JD, Segal ZV, Williams JM, Ridgeway VA, Soulsby JM, Lau MA (2000): Prevention of relapse/recurrence in major depression by mindfulnessbased cognitive therapy. J Consult Clin Psychol 68:615-623.

Van der Does AJW (2001): The effects of tryptophan depletion on mood and psychiatric symptoms. J Affect Disord 64:107-119.

Van der Does AJW, Booij L, Haffmans PMJ (2004): Cognitive and serotonergic vulnerability to depression: Convergent findings. Int J Neuropsychopharm 7(suppl 1):201

Williams JMG, Teasdale JD, Segal ZV, Soulsby J (2000): Mindfulness-based cognitive therapy reduces overgeneral autobiographical memory in formerly depressed patients. J Abn Psychol 109:150-155.

Young SN, Smith SE, Pihl RO, Ervin FR (1985): Tryptophan depletion causes a rapid lowering of mood in normal males. Psychopharmacol 87:173-177. 